\title{
COCOMPACTNESS IN ALGEBRA AND TOPOLOGY
}

\author{
GRAHAM J. LOGAN
}

(Received 28 July 1980)

Communicated by J. H. Rubinstein

\begin{abstract}
This paper discusses two notions, developed independently and both termed "cocompactness". The first arises in the area of topology, where J. de Groot and others' have studied spaces which are, in a certain sense, complementary to a given space. If the given space is compact then the complementary spaces are said to be cocompact. The second concept arises in the area of logic and general algebra. Loosely speaking a logic is compact if every inconsistent set of formulas has a finite inconsistent subset. This notion of compactness may be generalized to any closure algebra ${ }^{2}$ and the use of the term "cocompactness" to describe the generalization was suggested to the author by Dr. R. A. Bull.

It is shown here that topological and algebraic cocompactness are related in the following ways. Firstly, if a closure algebra is algebraically cocompact then its dual space is topologically cocompact, and conditions may be given for the implication to be reversible. ${ }^{3}$ Furthermore any cocompact topological space may be represented as the continuous 1-1 image of the dual space of a cocompact closure algebra. A final result relates another class of closure algebras with those topological spaces which are compact.
\end{abstract}

1980 Mathematics subject classifications (Amer. Math. Soc.): 03 G 24, 54 D 30.

The notation, results and references in [5], [6] and [7] are presupposed: in particular if $(X, C)$ is a closure algebra then $A \subseteq X$ is said to be consistent if and only if $C(A) \neq X, M_{X}$ is the family of maximal consistent subsets of $X, S$ : $P(X) \rightarrow P\left(M_{X}\right)$ is defined by $S(A)=\left\{\Delta \in M_{X}: A \subseteq \Delta\right\}$; and $\beta=\left\{S\left(A_{f}\right)\right.$ : $A_{f} \subseteq X, A_{f}$ finite $\}$ is the base for a topology $\tau$ on the dual space $\left(M_{X}, \tau\right) .(X, C)$ will be said to be (algebraically) cocompact if every inconsistent subset of $X$ has a finite inconsistent subset, and will be said to satisfy the Lindenbaum condition if every consistent set may be extended to a maximal consistent set.

\footnotetext{
${ }^{1} \operatorname{See}[1],[2],[4]$.

${ }^{2}$ See [3], page 13. tion.

OCopyright Australian Mathematical Society 1981
}

${ }^{3}$ Where the meaning is clear from the context I will use the term "cocompact" without qualifica- 
The following definitions are taken from [2]:

A closed base (closed subbase) for a $T_{3}$-space is a collection $\mathscr{B}$ of closed sets which form a neighbourhood base (subbase) for the topology. That is if $x$ lies in the open set $U$, there is a $B \in \mathscr{B}$ (a $\left.B=B_{1} \cap \cdots \cap B_{n} \in \mathscr{B}\right)$ such that $B \subseteq U$ and $x$ lies in the interior of $B$.

A $T_{3}$-space is cocompact if and only if it has a closed base $\mathscr{B}$ and every subfamily of $\mathscr{B}$ with the finite intersection property (f.i.p.) has a non-empty intersection.

The following theorem of A. Lindenbaum ([8], p. 98) will be required; it is a straightforward consequence of the definition of cocompactness and Zorn's Lemma.

THEOREM 1. If $(X, C)$ is a cocompact closure algebra then every consistent set may be extended to a maximal consistent set.

THEOREM 2. If $(X, C)$ is a cocompact closure algebra and $\left(M_{X}, \tau\right)$ its dual space then $\left(M_{X}, \tau\right)$ is a regular cocompact $T_{1}$-space.

Proof. In [5] it is shown that $\left(M_{X}, \tau\right)$ is a $T_{1}$-space, and in [6] that $\left\{S\left(A_{f}\right)\right.$ : $\left.A_{f} \in P(X)\right\}$ is a basis of clopen sets. It follows that $\left(M_{X}, \tau\right)$ is regular and that $\left\{S\left(A_{f}\right): A_{f} \in P(X)\right\}$ is a closed base. Hence to show that $\left(M_{X}, \tau\right)$ is cocompact it is only necessary to show that every subfamily of the base with the finite intersection property has a non-empty intersection. Suppose that $\Gamma=\left\{S\left(A_{f}\right)\right.$ : $f \in F\}$ has the f.i.p. and consider $A=\cup_{f \in F} A_{f}$. If $A_{g}$ is a finite subset of $A$ then we may choose $F^{\prime}$ a finite subset of $F$ so that $A_{g} \subseteq \cup_{f \in F^{\prime}} A_{f}$, and we then have $S\left(A_{g}\right) \supseteq S\left(\cup_{f \in F^{\prime}} A_{f}\right)=\bigcap_{f \in F^{\prime}} S\left(A_{f}\right) \neq \varnothing$. Hence there is some $\Delta \in M_{X}$ with $\Delta \in S\left(A_{g}\right)$, that is with $A_{g} \subseteq \Delta$ and so $C\left(A_{g}\right) \subseteq C(\Delta) \neq X$, showing that each finite subset of $A$ is consistent. Since $(X, C)$ is cocompact, $A$ is consistent. Furthermore we have by Theorem 1 that any consistent subset of a cocompact closure algebra may be extended to a maximal consistent subset. Hence there is some $\Delta \in M_{X}$ with $A \subseteq \Delta$ and so $\Delta \in S(A) \neq \varnothing$. But $\cap \Gamma=\cap_{f \in F} S\left(A_{f}\right)=$ $S\left(\cup_{f \in F} A_{f}\right)=S(A) \neq \varnothing$.

THEOREM 3. If $(X, C)$ is a closure algebra which satisfies the Lindenbaum condition and has a cocompact, $T_{3}$ dual space $\left(M_{X}, \tau\right)$ then $(X, C)$ is cocompact.

Proof. Suppose that every finite subset of $A \subseteq X$ is consistent, that is if $f \in F$ indexes the finite subsets of $A$ then $C\left(A_{f}\right) \neq X$ for each $f \in F$. Since $(X, C)$ satisfies the Lindenbaum condition we have that each $A_{f}$ is contained in a maximal consistent set and so $S\left(A_{f}\right) \neq \varnothing$ for each $f \in F$. It follows that the 
family $\left\{S\left(A_{f}\right): f \in F\right\}$ has the f.i.p., since if $F^{\prime}$ is a finite subset of $F$ then $\bigcap_{f \in F^{\prime}} S\left(A_{f}\right)=S\left(\cup_{f \in F^{\prime}} A_{f}\right) \neq \varnothing$ because $\bigcup_{f \in F^{\prime}} A_{f}$ is again a finite subset of $A$. Hence $\left\{S\left(A_{f}\right): f \in F\right\}$ is a subfamily of the base $\beta$ with the fi.p., and from the cocompactness of $\left(M_{X}, \tau\right)$ we may infer that $\cap_{f \in F} S\left(A_{f}\right) \neq \varnothing$. But $S(A)=$ $S\left(\cup_{f \in F} A_{f}\right)=\bigcap_{f \in F} S\left(A_{f}\right)$, and so there is some $\Delta \in M_{X}$ with $A \subseteq \Delta$, showing that $A$ is consistent and consequently that $(X, C)$ is cocompact.

THEOREM 4. If $Y$ is a cocompact $T_{3}$-space then $Y$ is the continuous, one-to-one image of the dual space of a cocompact closure algebra.

Proof. Since $Y$ is cocompact there is a closed subbase $X$ for the topology on $Y$. Define $C: P(X) \rightarrow P(X)$ by $C(A)=\left\{u \in X: \bigcap_{v \in A} v \subseteq u\right\}$. It is easily verified that $(X, C)$ is a closure algebra, and it is shown ${ }^{4}$ in [7] that if $\Gamma_{y}=\{u \in X: y \in u\}$, for each $y \in Y$, then $M_{X}=\left\{\Gamma_{y}: y \in Y\right\}$, and that if $\theta$ : $Y \rightarrow M_{X}$ is defined by $\theta(y)=\Gamma_{y}$ then $\theta$ is a 1-1 and onto function which preserves open sets. It follows that $\theta^{-1}: M_{X} \rightarrow Y$ is a continuous bijection, and it only remains to be shown that $(X, C)$ is cocompact.

Suppose that $A \subseteq X$ has every finite subset consistent, that is if $A_{f} \subseteq A$ then $C\left(A_{f}\right)=\left\{u \in X: \bigcap_{v \in A_{f}} v \subseteq u\right\} \neq X$. Then we must have $\cap_{v \in A_{f}} v \neq \varnothing$ for each $A_{f} \subseteq A$, so that $A$ is a subset of the subbase for $Y$ with the f.i.p. Since $Y$ is cocompact $\cap_{v \in A} v \neq \varnothing$ and we may choose $y \in \cap_{v \in A} v$. Since $Y$ is $T_{1}$, $Y \backslash\{y\}$ is an open set and so there is some $u^{*} \in X$ with $y \notin u^{*}$. Now $u^{*} \in X \backslash\left\{u \in X: \bigcap_{v \in A} v \subseteq u\right\}=X \backslash C(A)$, showing that $C(A) \neq X$. Consequently $A$ is consistent and $(X, C)$ is cocompact.

THEOREM 5. If $(X, C)$ is a closure algebra then the dual space $\left(M_{X}, \tau\right)$ is compact if and only if any subset of $X$ which intersects every member of $M_{X}$ has a finite subset which intersects every member of $M_{X}$.

Proof. Suppose that $\left(M_{X}, \tau\right)$ is compact and that $A \subseteq X$ is such that $A \cap \Delta$ $\neq \varnothing$ for each $\Delta \in M_{X}$. Then for each $\Delta \in M_{X}$ we may choose $A_{\Delta}$, a finite subset of $A \cap \Delta$. Since each $\Delta \in S\left(A_{\Delta}\right)$, and each $A_{\Delta}$ is finite, we have that $\left\{S\left(A_{\Delta}\right): \Delta \in M_{X}\right\}$ is an open over of $\left(M_{X}, \tau\right)$ and so there is some finite subcover $\left\{S\left(A_{\Delta_{i}}\right): 1 \leqslant i \leqslant n\right\}$. Now $\cup_{i=1} A_{\Delta_{i}}$ is a finite subset of $A$, and furthermore for each $\Delta \in M_{X}$ there is some $i, 1 \leqslant i \leqslant n$, with $\Delta \in S\left(A_{\Delta_{i}}\right)$, that is with $A_{\Delta_{i}} \subseteq \Delta$. This shows that $\cup_{i=1}^{n} A_{\Delta_{i}}$ is a finite subset of $A$ which intersects each maximal consistent set.

\footnotetext{
${ }^{4} \mathrm{It}$ is shown in [7] (Theorem 2) that these results hold for an open subbase $X$. However the proofs are also valid in the case where $X$ is a closed subbase. In particular the proof that $\theta$ preserves open sets can be carried over almost verbatim.
} 
On the other hand, suppose that $\left(M_{X}, \tau\right)$ is not compact. Then we may find an open $\operatorname{cover} \theta$ with no finite subcover, and we may refine $\theta$ to a $\operatorname{cover}\left\{S\left(A_{f}\right)\right.$ : $f \in F\}$ of members of the base that also has no finite subcover. Consider the family $\mathscr{F}=\left\{A_{f}: f \in F\right\}$, which has the property that for each finite subfamily there is some $\Delta \in M_{X}$ such that every member of the subfamily is not a subset of $\Delta$, (note that if $F^{\prime} \subseteq F$ indexed a finite subfamily that did not possess this property then $\left\{S\left(A_{f}\right): f \in F^{\prime}\right\}$ would be a finite subcover of $\left.\left\{S\left(A_{f}\right): f \in F\right\}\right)$. Call this property $P$ and let $\Gamma$ be the class of all families that have the property $P$ and that contain $\mathscr{F}$. Since $\mathscr{F} \in \Gamma, \Gamma \neq \varnothing$; and if $\left\{\mathscr{F}_{k}: k \in K\right\}$ is a chain of families in $\Gamma$ then it is clear that $\cup_{k \in K} \mathscr{F}_{k}$ also has the property $P$. This shows that each chain in $\Gamma$ has an upper bound and so we may appeal to the axiom of choice in the form of Zorn's lemma to establish the existence of a maximal family $\mathscr{F}^{*}$. Suppose that for $x_{1}, x_{2} \in X,\left\{x_{1}\right\} \notin \mathscr{F}^{*},\left\{x_{2}\right\} \notin \mathscr{F}^{*}$, then by the maximality of $\mathscr{F}^{*}$ there are two finite families $\mathscr{F}_{1}, \mathscr{F}_{2} \in \mathscr{F}^{*}$ and we have for each $\Delta \in M_{X}$, that $\Delta$ has as a subset some member of $\left\{\left\{x_{1}\right\}\right\} \cup \mathscr{F}_{1}$ and also some member of $\left\{\left\{x_{2}\right\}\right\} \cup \mathscr{F}_{2}$. Hence each $\Delta$ has as a subset some member of $\left\{\left\{x_{1}, x_{2}\right\}\right\} \cup \mathscr{F}_{1} \cup \mathscr{F}_{2}$. This shows that $\left\{x_{1}, x_{2}\right\} \notin \mathscr{F}^{*}$. By using a simple induction and contraposition we may infer that if $A_{f} \in \mathscr{F}^{*}$ then there is some $x \in A_{f}$ with $\{x\} \in \mathscr{F}^{*}$. Put $W=\left\{x: x \in A_{f} \in \mathscr{F}^{*},\{x\} \in \mathscr{F}^{*}\right\}$. Since $\mathscr{F}^{*} \supseteq \mathscr{F}$ and $\left\{S\left(A_{f}\right): A_{f} \in \mathscr{F}\right\}$ is a cover of $M_{X}$, we have that for each $\Delta \in M_{X}$ there is some $A_{f} \in \mathscr{F}^{*}$ with $A_{f} \subseteq \Delta$, so that there is some $x \in W$ with $x \in \Delta$ and now $W$ intersects every maximal consistent set. On the other hand if $V$ is a finite subset of $W$ then $\{\{x\}: x \in V\}$ is a finite subfamily of $\mathscr{F}^{*}$ and so there is some $\Delta \in M_{X}$ that contains no $\{x\}$ for $x \in V$. That is no finite subset of $W$ intersects every maximal consistent set.

The author expresses his thanks to the referee for helpful advice on presentation.

\section{References}

[1] J. M. Aarts, J. de Groot, R. H. McDowell, 'Cotopology for Metrizable Spaces', Duke Math. J. 37 (1970), 291-295.

[2] J. M. Aarts, J. de Groot, R. H. McDowell, 'Cocompactness', Nietw Arch. Wisk. (3) 28 (1970), 2-15.

[3] D. J. Brown and R. Susko, 'Abstract Logics', Dissertationes Math. 102 (1973), 9-41.

[4] J. De Groot, 'Subcompactness and the Baire Category Theorem', Indag. Math. 25 (1963), 761-700.

[5] G. J. Logan, 'Closure Algebras and $T_{1}$-spaces’, Z. Math. Logik Grundlagen Math. 23 (1977), 91-92.

[6] G. J. Logan, 'Closure Algebras and Boolean Algebras', Z. Math. Logik Grundlagen Math. 23 (1977), 92-93. 
[7] G. J. Logan, 'On the Representation of Metric Spaces', Bull. Austral Math. Soc. 20 (1979), 367-375.

[8] A. Tarski, 'Fundamental Concepts of the Methodology of the Deductive Sciences', Reprint in Logic, Semantics, Metamathematics (Oxford University Press (1956), 60-109).

Department of Applied Sciences

Christchurch Polytechnic

New Zealand 